\title{
Civilisations
}

Revue internationale d'anthropologie et de sciences

humaines

$57 \mid 2008$

Tourisme, mobilités et altérités contemporaines

\section{Conflits et enjeux identitaires dans le tourisme rural à Yangshuo, Chine}

\section{Timothy Leicester}

\section{OpenEdition}

\section{Journals}

Édition électronique

URL : http://journals.openedition.org/civilisations/1370

DOI : 10.4000/civilisations. 1370

ISSN : 2032-0442

\section{Éditeur}

Institut de sociologie de l'Université Libre de Bruxelles

\section{Édition imprimée}

Date de publication : 29 décembre 2008

Pagination : 223-241

ISBN : 2-87263-022-8

ISSN : 0009-8140

Référence électronique

Timothy Leicester, "Conflits et enjeux identitaires dans le tourisme rural à Yangshuo, Chine »,

Civilisations [En ligne], 57 | 2008, mis en ligne le 29 décembre 2011, consulté le 10 décembre 2020.

URL : http://journals.openedition.org/civilisations/1370; DOI : https://doi.org/10.4000/civilisations.

1370

(c) Tous droits réservés 


\title{
Conflits et enjeux identitaires \\ dans le tourisme rural à Yangshuo, Chine ${ }^{1}$
}

\author{
Timothy LEICESTER
}

\begin{abstract}
Résumé : Yangshuo, ville du Guangxi dans le sud-ouest de la Chine, est emblématique de l'essor récent du tourisme chinois. Située dans des paysages spectaculaires célébrés par les voyageurs lettrés depuis des siècles, cette agglomération rurale attire aujourd'hui des centaines de milliers de visiteurs chinois et étrangers. Lieu de rencontre entre sociétés urbaines et rurales, elle donne à voir une série d'inégalités de la société chinoise contemporaine. Le tourisme y génère de nombreux conflits autour de l'appropriation des ressources, l'interprétation des sites, et la représentation de la campagne et de la paysannerie. Cet article présente le tourisme chinois contemporain dans le contexte historique et culturel qui lui est propre, et met en relief certaines spécificités des pratiques touristiques chinoises. À travers cela, le texte met en cause nos manières de penser le tourisme, et révèle des changements sociaux à l'œuvre dans la Chine contemporaine.
\end{abstract}

Mots-clés : tourisme chinois, regard touristique, inégalités ville-campagne, mobilité, sites touristiques.

\begin{abstract}
The town of Yangshuo in Guangxi, south west China, is emblematic of the recent rise of Chinese tourism. Situated in a spectacular landscape celebrated by generations of literary travelers, this rural town now attracts hundreds of thousands of Chinese and international visitors. As a meeting place of urban and rural society, the town shows several inequalities of contemporary Chinese society. Tourism in Yangshuo also generates numerous conflicts concerning the appropriation of resources, the interpretation of tourist sites, and the representation of the countryside and rural society. The present case study allows us to consider contemporary Chinese tourism in relation to its own historical and cultural context, and brings to light certain specificities of Chinese touristic practices. The article furthermore reconsiders the common ways in which we think about tourism, and reveals social changes currently taking place in China.
\end{abstract}

Key words : Chinese tourism, tourist gaze, urban-rural inequality, mobility, tourist spots.

1. Cet article s'appuie sur une enquête de terrain d'une durée de trois mois menée en 2007 à Yangshuo, financée par le Centre des Études sur la Chine Moderne et Contemporaine (CECMC) de l'EHESS, Paris. Par ailleurs l'auteur visite régulièrement la ville de Yangshuo dans le cadre de son travail comme accompagnateur touristique pour une agence spécialisée dans les voyages en Chine. 


\section{L} croissance fulgurante du tourisme chinois au cours des vingt dernières années est une des transformations majeures du paysage touristique mondial. D'ici dix ans, la Chine deviendra le plus grand pays producteur de touristes internationaux (CLSA 2005) mais déjà, le tourisme et les touristes chinois sont indiscutablement "parvenus à l'âge mûr » (Chan 2006). Cette tendance forte nous oblige à revisiter et à reconsidérer les approches employées pour l'analyse du tourisme qui sont principalement basées sur l'expérience des touristes occidentaux. Si cela est vrai pour le tourisme international, cela l'est d'autant plus pour le tourisme à l'intérieur de la Chine où les touristes domestiques représentent plus de 95\% du nombre total des touristes (CNTA 2008).

La Chine nous offre la possibilité de mettre à l'épreuve certaines hypothèses concernant le tourisme. Au premier abord l'exemple de la Chine semble confirmer le constat selon lequel le tourisme est essentiellement un phénomène moderne et occidental (Cohen 1994 : 12) et l'émergence du tourisme non-occidental la reproduction d'un même phénomène sous l'influence de la mondialisation. La circulation des biens, des idées, des signes et des personnes qui est constitutive de la mondialisation est certainement l'un des facteurs qui a stimulé le tourisme en Chine, sans parler de l'impulsion donnée par des institutions internationales telles que l'UNESCO ou l'Organisation Mondiale du Tourisme. D'autre part, le contexte sociologique de l'essor du tourisme domestique en Chine est largement similaire à celui déjà identifié en Occident comme étant constitutif du tourisme moderne : l'émergence d'une classe moyenne, l'ordonnance du temps de travail et de loisir, l'amélioration des transports (Urry 1990). Comme le fait remarquer Olivier Evrard, il est pourtant problématique, de considérer les touristes non occidentaux en général, et chinois en particulier, comme de simples « avatars tardifs de leurs équivalents occidentaux » (Evrard 2006 : 156). Les pratiques et le « regard» des touristes chinois doivent autant à des spécificités historiques et culturelles chinoises qu'aux pratiques ou à des schémas de pensée occidentaux. Ce qui relève du moderne doit être mis en relation avec la constitution d'une modernité proprement chinoise, et ce qui relève de la mondialisation est à considérer à la lumière des réseaux transnationaux qui la constituent et notamment du rôle important joué par la diaspora chinoise. À cet égard, le tourisme contemporain est une sorte d'instantané qui capture un moment du voyage qu'entreprend ce pays vers sa propre modernité, ouvert sur l'Asie et sur le monde, mais ancré dans une réalité et un passé propres.

Il y a dix ans déjà, Tim Oakes constatait que le tourisme était devenu «l'un des théâtres importants où se joue la problématique de la modernité chinoise » (Oakes 1998 : 47). Depuis, les chercheurs sont de plus en plus nombreux à y prêter attention. C'est particulièrement le cas des anthropologues travaillant sur les minorités ethniques pour lesquelles le tourisme est devenu un élément si important dans la constitution et la représentation de leur identité qu'il est désormais impossible d'en faire abstraction (voir Schein 1997; McKhann 2001; Gladney 2004; Notar 2006 et l'ouvrage collectif coédité par Tan Chee-Beng 2001). En dehors de ces cas où le tourisme s'est en quelque sorte imposé à l'anthropologue, d'autres chercheurs ont étudié l'établissement de sites et de parcs d'attractions (Anagnost 1997; Nyìri 2006; Breidenbach et Nyìri 2007 et David 2007) ou le développement du tourisme chinois international (Chan 2006 et 
Nyìri 2005). Ces travaux commencent à défricher ce vaste et complexe champ d'étude qui est extrêmement révélateur quant à l'évolution de la société chinoise, notamment en ce qui concerne l'identité nationale et l'autorité culturelle de l'État.

Yangshuo présente le double intérêt d'être un des sites les plus emblématiques du tourisme chinois contemporain alors qu'il fait également partie des sites canoniques du tourisme lettré pré-moderne. Il nous permet ainsi d'approfondir notre perspective historique du phénomène touristique en Chine. En étudiant les changements sociaux induits par le tourisme dans cette localité rurale, nous pourrons éclairer des processus plus généraux à l'œuvre dans l'évolution sociale de la Chine, ainsi que les inégalités et rapports de pouvoir qui les sous-tendent.

\section{La mobilité et le tourisme : inégalités ville / campagne}

Le tourisme domestique en Chine doit être étudié à la lumière de la mobilité croissante de la société chinoise. On estime à 1,6 milliard le nombre de " voyages » domestiques en 2007 (CNTA 2008). Cependant, ce chiffre global cache d'importantes distinctions quant aux profils des voyageurs et à leurs motifs de voyage. Voyages d'affaires, réunions familiales, flux migratoires, et voyages de loisir sont ici tous confondus. D'autre part, ce chiffre masque d'importantes inégalités entre la mobilité des citadins et celle des ruraux (la majorité de la population chinoise). Certes, le nombre de voyageurs ruraux dépasse celui des voyageurs citadins ( 1 milliard contre 600 millions), mais les citadins sont $60 \%$ plus mobiles, et dépensent quatre fois plus par personne lors de leurs déplacements (Ibid.). En effet, une grande partie des voyageurs ruraux se déplacent des campagnes vers les villes dans le cadre de la migration économique, ou, en sens inverse pour rejoindre lors des fêtes leurs familles restées au village. Parmi les urbains, et bien que certains déplacements se fassent dans le cadre de la migration économique (notamment des villes moyennes vers les grandes métropoles), la plupart relèvent du cadre des loisirs. Ces différentes formes de mobilité témoignent des inégalités sociales et économiques, notamment entre ville et campagne.

La disparité entre ville et campagne est l'une des caractéristiques marquantes de la société chinoise depuis les années 1950 (Cheng et Selden 1994 : 667). Elle résulte d'un système administratif qui classifie la population comme « urbaine » ou « rurale » selon le lieu d'origine, et exclut les ruraux des protections sociales accordées aux citadins, ainsi que d'une politique qui a favorisé le développement urbain (Naughton 1995 : 73). En matière d'éducation, de revenus, de santé ou de mobilité sociale, le citadin chinois est privilégié par rapport à ses compatriotes d'origine rurale.

En provoquant la rencontre de ces deux populations, le tourisme rural met en scène et reproduit de manière frappante ces inégalités. Nous pouvons observer des similitudes avec d'autres formes de tourisme qui mettent en relation des populations issues de sociétés au niveau de développement différent. Néanmoins, le tourisme rural en Chine est particulier dans la mesure où il met en jeu des inégalités au sein d'une même société. Ainsi, pour les visiteurs (les touristes urbains), les visités (les habitants ruraux) ne sont pas simplement « autres » mais également leurs doubles. Le tourisme rural en Chine se présente alors comme un jeu de miroirs, où le regard que portent les touristes urbains sur l'espace et les habitants ruraux participe aussi du regard qu'ils portent sur eux-mêmes et sur leur pays. Pour comprendre ce double regard, il est nécessaire de retracer brièvement l'histoire du tourisme en Chine et le rôle important de l'État dans son développement récent. 


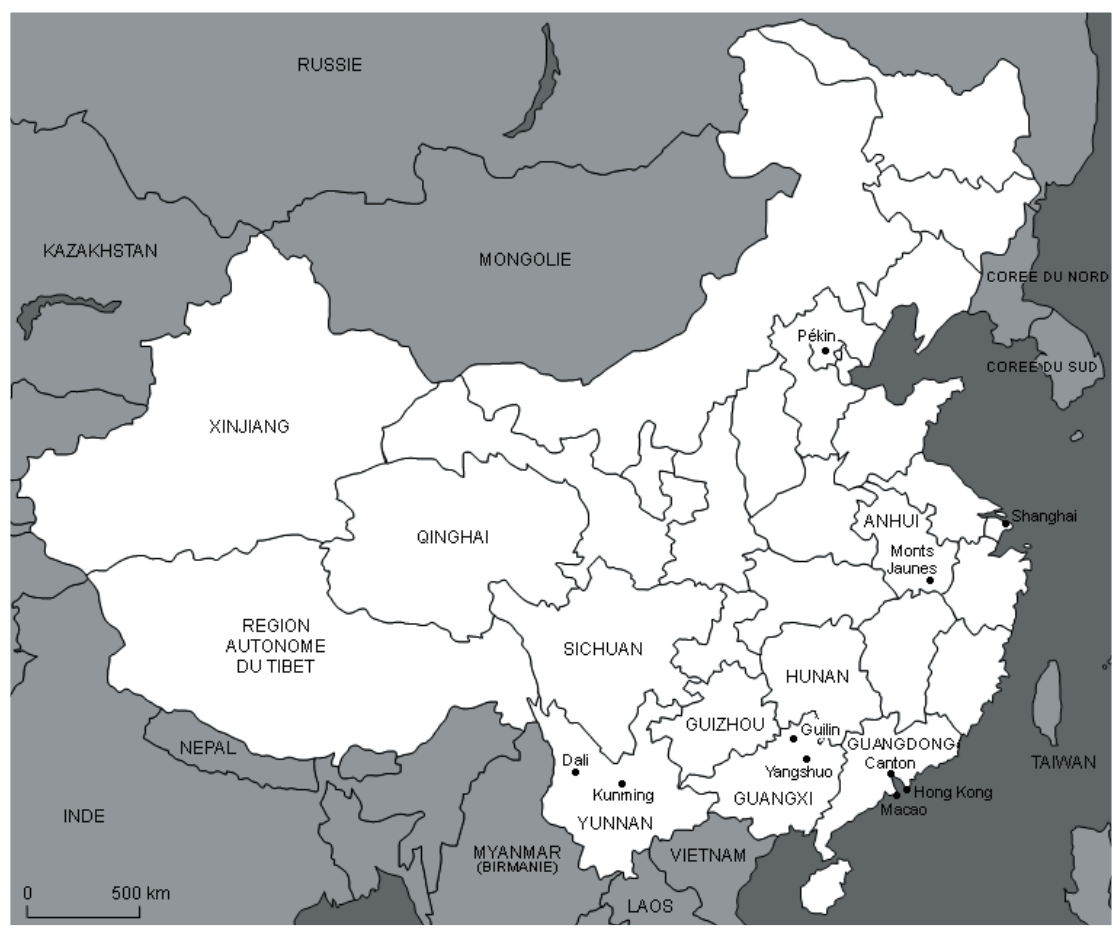

La Chine et les sites mentionnés dans l'article @ http://histgeo.ac-aix-marseille.fr

\section{Le développement du tourisme et le rôle de l'État}

La Chine a une longue tradition de tourisme lettré dont les origines remontent aux premiers siècles de notre ère, mais qui s'est développée surtout à partir du $16^{\mathrm{e}}$ siècle avec la croissance du commerce et de la mobilité dans la société chinoise (Brook 1998 et Zhang 2004). Cette tradition valorisait avant tout l'appréciation de scènes paysagères choisies et la visite de sites pittoresques consacrés par la tradition littéraire et picturale. En se rendant sur ces sites, le voyageur lettré pouvait se mettre en relation avec la tradition culturelle et littéraire et ainsi affirmer son appartenance à une élite sociale. C'était aussi un processus par lequel des territoires marginaux étaient intégrés au système-monde chinois (Strassberg $1994: 8$ ).

Le tourisme a été critiqué durant l'ère maoïste pour son caractère bourgeois. Mais lorsqu'il renaît progressivement à partir de 1973, c'est quand même en référence à la tradition du tourisme lettré que les sites d'intérêt et la manière de les mettre en valeur furent désignés. La revalorisation du concept chinois de mingsheng ou « site pittoresque célèbre " constitua un élément important de cette renaissance touristique. Ce terme, utilisé autrefois pour désigner les sites d'intérêt dans le tourisme lettré, a été repris au compte de l'État qui a désigné un nouveau canon de sites touristiques essentiellement basé sur ceux qu'avait valorisés la tradition lettrée. 
C'est également dans cette tradition lettrée qu'on a puisé pour revaloriser le tourisme et pour reconstituer un répertoire de pratiques touristiques. On assiste par exemple dans les années 1980 à la publication de nombreuses compilations de récits de voyages classiques (youji) rebaptisés « littérature touristique » (lüyou wenxue). Des revues touristiques qui apparaissent à la même époque consacrent également de nombreux articles aux pratiques touristiques des lettrés (Nyìri $2006: 8$-12) et les guides touristiques de cette époque reprennent la forme et le contenu des guides classiques destiné aux voyageurs lettrés. Cette réhabilitation du tourisme lettré a largement influencé la manière dont les touristes chinois conçoivent le tourisme, notamment en ce qui concerne le type de site visité. Lors d'un sondage réalisé en 1997, 62\% des personnes interrogées considéraient que seul un mingsheng pouvait constituer un site digne d'intérêt touristique (Niquet 1998 : 7). Pour l'État, la revalorisation du concept de mingsheng avait un double objectif. D'une part, c'était une manière d'ancrer le tourisme dans une tradition nationale, d'autre part c'était un moyen de mieux contrôler tant les flux et les revenus du tourisme que son contenu idéologique.

C'est surtout à partir de 1979 que le tourisme se développe de manière significative. Cette année-là, Deng Xiaoping, grimpant les Monts Jaunes (Huangshan, site majeur du tourisme lettré), a déclaré sa volonté de faire de la Chine « une puissance mondiale du tourisme» (Niquet 1998: 1). Dans la décennie suivante, le nombre de touristes internationaux visitant la Chine a augmenté progressivement. Dans un premier temps, il s'agissait de faire rentrer des devises étrangères dans le pays et de renvoyer une image impressionnante de la Chine vers l'extérieur. Les paysages spectaculaires des lieux comme la rivière Li (Lijiang) à Yangshuo, que nous étudierons ci-dessous, ou les Monts Jaunes, devaient évoquer l'esprit national et la grandeur de la Chine. Ils se prêtaient particulièrement bien à cette nouvelle conception nationaliste du mingsheng en raison de leur nature manifestement séculaire ${ }^{2}$. De plus, ils étaient tous les deux associés à des mouvements de résistance nationale dans le passé ${ }^{3}$, donc davantage en accord avec l'idéologie du Parti communiste.

En exerçant un contrôle sur la désignation des sites touristiques, l'État pouvait également contrôler l'image du pays qu'ils véhiculent. Ce pouvoir était renforcé par le monopole les agences de voyage d'État et par la création de joint-ventures, des partenariats entre des investisseurs étrangers et des entreprises d'État ou des organes des gouvernements locaux. Par ce biais, l'État pouvait attirer les investissements nécessaires à l'amélioration des infrastructures touristiques tout en gardant une main mise sur leur évolution et une partie des bénéfices.

Au cours des années 1990, la dérégulation du secteur touristique permit à d'autres acteurs de s'y impliquer, entraînant une diversification des pratiques. Le tourisme domestique connut également une forte croissance suite à l'instauration de la semaine

2. À la différence de certains autres mingsheng qui étaient également des sites de pèlerinage bouddhistes ou taoïstes, la rivière Li et les Monts Jaunes avaient depuis longtemps été « sécularisés » par le tourisme lettré.

3. Les Monts Jaunes avaient servi de base pour un mouvement anti-Manchu suite à l'établissement de la dynastie Qing (Cahill 1992: 275) et Guilin et Yangshuo étaient associés à la résistance antijaponaise dans les années 1930 et 1940 (Hutchings 1986). 
de travail de cinq jours en 1995 et à la prolongation des vacances nationales en 1999. Le tourisme est alors de plus en plus perçu comme un vecteur civilisateur et modernisateur. On promeut le tourisme ethnique et le tourisme rural comme des moyens de développer les économies des régions sous-développées ou encore pour «jeter la lumière de la civilisation moderne sur chaque recoin rural » (Wu 1997 cité par Breidenbach et Nyìri 2007 : 325). Le rôle du tourisme dans le développement rural est particulièrement mis en valeur depuis l'arrivée à la tête du gouvernement en 2003 d'une nouvelle génération de dirigeants qui se veulent plus sensibles aux problèmes des campagnes (Kahn 2004). Cependant, entre la volonté des gouvernements nationaux et locaux de garder un contrôle sur les revenus et sur l'interprétation des sites touristiques d'une part, et la volonté d'en faire profiter les habitants ruraux d'autre part, il y a souvent des contradictions.

\section{L'évolution du tourisme à Yangshuo}

Yangshuo, chef-lieu du district du même nom, se trouve dans la Région autonome zhuang de Guangxi dans le sud-ouest de la Chine ${ }^{4}$. Le développement touristique dans et autour de cette petite ville reflète clairement l'histoire du tourisme, les différentes politiques d'État le concernant et les contradictions et conflits d'intérêts qui en découlent. Ce site attire des visiteurs depuis des siècles en raison de ses paysages karstiques spectaculaires. Bien que Yangshuo soit moins célèbre que la ville voisine de Guilin, les premiers écrits sur ses paysages remontent néanmoins au $9^{\mathrm{e}}$ siècle (Wang 2006 : 75). Yangshuo se situe sur la rivière Li, 83 kilomètres en aval de Guilin, rivière navigable qui, jusqu'au $20^{\mathrm{e}}$ siècle, était une des voies de communication principales entre le Centre et le Sud de la Chine. Le district de Yangshuo connut un commerce florissant au fil des siècles, et fut une base importante pour l'implantation de la culture et de l'autorité politique chinoises dans la région.

Guilin et, à un moindre degré, Yangshuo, constituaient des sites canoniques de la tradition du tourisme lettré. Dans les deux villes il existait, depuis des siècles, des circuits touristiques établis composés de scènes et de sites paysagers choisis ${ }^{5}$. Ces derniers étaient hautement aménagés avec kiosques, inscriptions, temples et de fortes connotations littéraires. Au début du $20^{\mathrm{e}}$ siècle, la réputation des deux villes est également promue par les visites de personnalités politiques importantes, et l'installation de célèbres artistes et écrivains.

Guilin et Yangshuo sont parmi les premiers lieux à être rouverts aux touristes étrangers en 1973. La rivière Li est également un des premiers sites à être désigné comme mingsheng en 1982. Dans un premier temps, les visiteurs sont en majorité des touristes étrangers, dont une partie importante vient de Hong Kong et de Macao (Xu 1999: 61). Les circuits touristiques étaient restreints à un nombre limité de sites encadrés par les autorités locales, principalement ceux prisés par la tradition lettrée. Outre ces sites, un des attraits principaux était, et reste encore aujourd'hui, la croisière sur la rivière Li de

4. Le territoire chinois est composé de 22 provinces, 4 municipalités, 2 régions administratives spéciales (Hong Kong et Macao) et 5 régions « autonomes » (zizhi qu), dont le Guangxi.

5. Ces circuits, établis par des lettrés locaux ou par des voyageurs, étaient souvent composés de « huit scènes paysagères » (ba jing) considérées comme incontournables pour l'appréciation du lieu et étaient largement reprises et citées par les voyageurs antérieurs. Concernant Yangshuo voir notamment Xu (1920 [1673]). 
Guilin jusqu'à Yangshuo. Une fois arrivé à Yangshuo, on traversait la ville à pied jusqu'au car pour regagner Guilin, s'arrêtant en route dans quelques sites pittoresques choisis. Ainsi enclavé, le tourisme concernait très peu les habitants de Yangshuo, à part quelques marchands de souvenirs installés près de la jetée d'arrivée des bateaux de croisières et les quelques villages situés près des sites.

Au cours des années 1980, la ville est repérée par des touristes indépendants occidentaux, et mentionnée dans des guides tels que Lonely Planet. Comme certains autres lieux recommandés dans ce guide, tel que la ville de Dali dans la province voisine de Yunnan (Notar 2006 : 20-46), Yangshuo devient l'un des lieux de prédilection des backpackers. Plusieurs hôtels, restaurants et cafés proposant des menus adaptés aux goûts occidentaux ont été ouverts par des entrepreneurs locaux pour répondre à leurs besoins. Ces commerces sont concentrés dans un périmètre restreint le long de la rue de l'Ouest, axe principale de la veille ville, qui est surnommée par les habitants locaux la « rue des étrangers » (Yangren jie) en raison du grand nombre de touristes étrangers qui s'y trouvent ${ }^{6}$.

C'est surtout à partir du milieu des années 1990 que le tourisme domestique se développe à Yangshuo. Alors que le nombre de touristes étrangers est resté stable, variant entre 25000 et 50000 par an, entre 1985 et 2004, le nombre de touristes chinois est passé de quasiment zéro à 512000 par an au cours de la même période. Et cela, sans compter les 1,75 million de touristes qui débarquent à Yangshuo après la croisière sur la rivière Li et visitent les sites alentour, sans dormir sur place (WTO 2005).

Désormais les touristes étrangers sont une minorité à Yangshuo, et les infrastructures se réorientent vers les besoins de cette nouvelle clientèle chinoise. Les différences de pratiques entre ces deux groupes de touristes sont caractéristiques des divergences de leur « regard ». Pour les touristes occidentaux, héritiers du « regard romantique » (Urry 1990 : 45), Yangshuo avec ses campagnes de rizières et de montagnes, peuplées de paysans en chapeau de paille, représente l'idéal de la Chine immobile et éternelle. Rêvant de solitude et du sublime de la nature sauvage, ils explorent les campagnes en VTT, font de l'escalade dans les collines, et descendent la rivière en canoë à la recherche d'expériences « authentiques ». Les touristes chinois, héritiers de la tradition lettrée, se déplacent en groupe pour visiter les sites aménagés, admirer des paysages spécifiques mis en valeur par les guides, et rêvent d'une nature non pas sauvage mais embellie par l'intervention humaine. Ces divergences confirment les observations d'autres anthropologues selon lesquelles le tourisme du regard romantique concerne toujours le corps alors que «le tourisme chinois, concerne, toujours, l'observation des scènes désignées comme étant "correctes" » (Breidenbach et Nyìri 2007 : 328).

Bien que le tourisme soit ancré dans une tradition historique, il est pourtant vécu par les Chinois comme une activité hautement moderne. Pour les classes moyennes urbaines, le tourisme est une forme de consommation ostentatoire qui «n'est pas qu'un loisir mais une forme de participation active à la modernité » (Nyìri $2006: 84$ ). Faire du tourisme, c'est aussi montrer sa volonté d'accéder aux formes de loisirs déjà généralisées dans les pays développés. C'est pourquoi la présence de touristes étrangers à Yangshuo est devenue l'un des attraits de la ville mis en valeur par les autorités locales qui se vantent

6. Beth Notar remarque le développement d'une rue similaire dans la ville de Dali (Notar $2006: 28$ ). 
d'être un "global village » (diqiu cun) où " les traditions culturelles orientales et la civilisation moderne occidentale rentrent en contact» (Wang 2004 : 69). Dans « la rue des étrangers », les touristes chinois, désormais bien plus nombreux que les Occidentaux, se ruent sur ces derniers pour se faire prendre en photo et échanger quelques mots en anglais. Des écoles de langue anglaise ont également ouvert dans la ville et attirent des centaines d'étudiants venus de partout dans le pays, tous désireux de rentrer en contact avec la population touristique cosmopolite. Ici, comme à Dali, où les touristes occidentaux sont également devenus des objets du tourisme, le « regard touristique » s'est retourné sur eux-mêmes. (Notar $2006: 43$ )

\section{Conflits et inégalités à Yangshuo}

Le tourisme domestique joue aujourd'hui un rôle beaucoup plus important que le tourisme international dans le développement du district. Les pratiques spécifiques aux touristes domestiques ont par ailleurs des implications particulières sur l'évolution et l'impact du tourisme sur la société locale, des tendances qui sont clairement visibles dans les campagnes autour de Yangshuo.

Un des premiers villages qui a su tirer des bénéfices du tourisme à Yangshuo est Chuanyan, situé à sept kilomètres de la ville. Situé près d'une rivière et entouré de montagnes, ce village pittoresque doit sa réputation à la présence d'un très grand et ancien arbre banian qui, outre sa taille impressionnante, a servi de décor dans une scène du film musical Liu Sanjie (" Troisième sœur Liu ») réalisé par Su Li en 1960. Le scénario de ce film très populaire, s'inspire d'une légende des Zhuang, la plus importante des 55 « nationalités minoritaires » (shaoshu minzu) officiellement reconnues par les autorités chinoises ${ }^{7}$. Ironiquement, si le Guangxi est désigné comme Région autonome zhuang, les populations zhuang de Yangshuo ont depuis plusieurs siècles été repoussées ou assimilées par l'implantation de populations han (la « nationalité » majoritaire en Chine).

Dans ce film, une chanteuse célèbre se range aux côtés des paysans pour combattre les exactions d'un propriétaire terrien. Le scénario reflète l'idéologie maoïste de son époque. En utilisant les paysages de Yangshuo comme décor, il les inscrivait dans une tradition révolutionnaire, affirmant que ces paysages, comme le pays, appartiennent au peuple et non aux élites lettrées. Il fait partie d'un certain nombre de productions réalisées à cette époque qui mettaient en scène la vie des diverses «nationalités minoritaires » en Chine (Notar 2006 : 50). Beaucoup de ces films décrivaient les dures conditions de vie avant la révolution communiste pour souligner les bienfaits de la période postrévolutionnaire. Ils servaient à intégrer ces zones frontalières et culturellement hétérogènes dans l'imaginaire nationale. L'utilisation de personnages issus de ces «minorités » exotiques permettait également d'explorer des thèmes romantiques et érotiques qui auraient été jugés choquants s’ils avaient concerné des personnages de la majorité han (Gladney 2004 : 77).

7. Sur la constitution de cette population et les enjeux politiques et identitaires suscités par sa désignation comme une « nationalité minoritaire » voir l’ouvrage de Katherine Palmer Kaup (2000). 


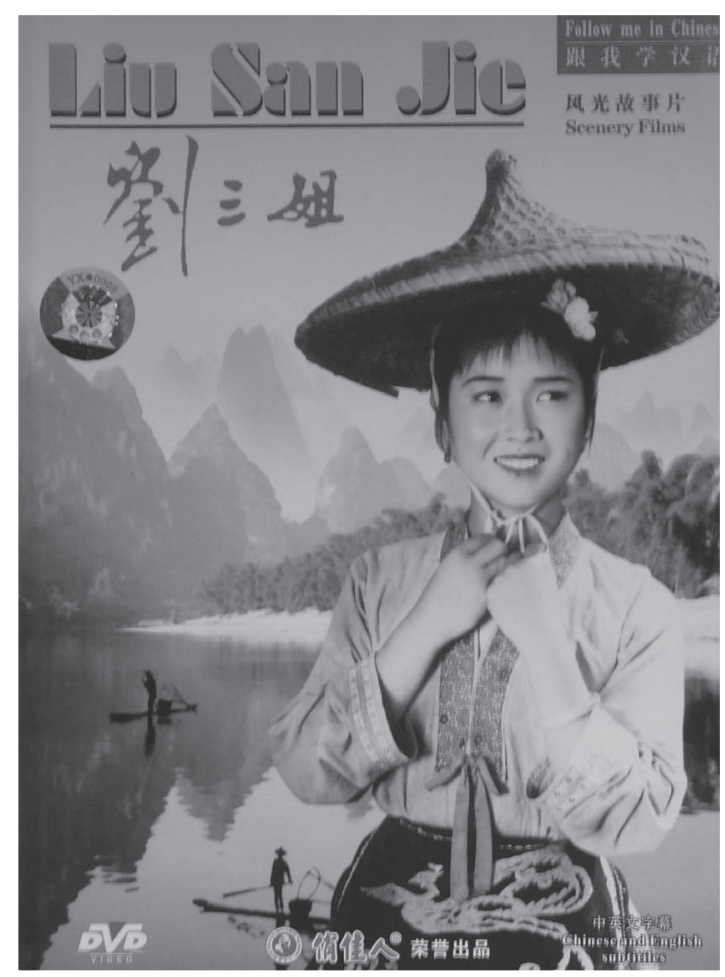

Jaquette de la réédition en DVD du film Liu Sanjie @ C Changchun Dianying Zhipianchang [1960].

Pour les autorités locales, il s'agissait d'un site tout à fait adapté au concept de mingsheng promu par les autorités centrales. Par contre, pour les 480 habitants du village, l'arbre avait un tout autre symbolisme car il est considéré par eux comme l'incarnation d'une divinité nourricière et protectrice du village et de la rivière. Lors des fêtes, ou lorsque quelqu'un souhaite par exemple de l'aide pour passer des examens, guérir une maladie, ou avoir un fils, on brûle de l'encens et on colle des offrandes en papier au pied de l'arbre.

À l'arrivée des premiers touristes, à la fin des années 1970, les villageois demandaient quelque maos $\left(0.1\right.$ Yuan $\left.^{8}\right)$ pour traverser leurs terres et accéder au pied du banian. Le site attirait des touristes de Hong Kong et de Macao, familiers du film Liu Sanjie, et quelques touristes occidentaux, séduits par les paysages du site sans connaître la référence du film. Les paysans ont utilisé une partie de cet argent pour aménager un chemin reliant l'arbre à la route qui passe près du village.

Une nouvelle phase dans l'aménagement du site du banian a commencé en 1982, lorsque le gouvernement local de Yangshuo s'est approprié les terres autour de l'arbre en ne donnant qu'une maigre compensation aux paysans qui les cultivaient. Les expropriations de terres agricoles, extrêmement récurrentes en Chine, sont l'une des sources majeures

8. Un Yuan équivaut à 10 centimes d'euro. 
de conflit entre paysans et gouvernements locaux (Guo 2001 et Notar 2006 : 132 ). Elles sont facilitées par l'ambiguïté des lois concernant la propriété dans les campagnes (Ho 2001). Si chaque famille paysanne cultive ses propres terres depuis la décollectivisation de l'agriculture en 1978, elle ne possède qu'un droit d'usufruit sur ces biens qui restent propriété d'État (Thireau et Wang 2001 : 15). Au total, un quart de la superficie totale des terres cultivables du village a été exproprié par le gouvernement au cours des années 1980, réduisant de manière considérable l'autonomie économique du village. En plus des paiements initiaux, le gouvernement donne chaque année une somme de quelques dizaines de milliers de Yuans en compensation au comité villageois, qui est utilisée pour les travaux collectifs (irrigation, entretien des routes etc.).

Le bureau des parcs et des jardins de Yangshuo, qui a pris en charge le développement du site, baptisé « le parc du grand banian » (Darongshu gongyuan), a réaménagé le terrain autour de l'arbre en jardin, construit un bâtiment au bord de la route pour vendre des billets d'entrée, et un grand parking pour accueillir les cars touristiques en provenance de Yangshuo. Les bénéfices du parc sont versés au gouvernement local qui, depuis les réformes initiées dans les années 1980, doit trouver ses propres sources de revenus et perçoit le tourisme comme une manne propice. La manière dont le gouvernement s'est approprié ce site est caractéristique de la politique nationale envers le tourisme et de la volonté de contrôler l'interprétation des sites. Par ailleurs, elle reflète l'héritage $\mathrm{du}$ tourisme lettré en ce qui concerne le rapport au paysage, où ce n'est pas l'aspect « naturel » d'un site qui prime comme c'est le cas dans le mythe occidental de la nature sauvage, mais son aménagement et embellissement par la mise en évidence de références culturelles et par l'art paysager.

Selon les autorités de Yangshuo, les paysans ne disposaient pas des capacités nécessaires pour l'entretien et l'aménagement d'un site touristique. Cependant, 25 ans après, les habitants de Chuanyan continuent à exprimer leur regret que l'arbre dont ils se sont occupés «depuis des générations » ne leur appartienne plus. Ils continuent à revendiquer : « nous n'avons pas vendu ces terres, elles ont été expropriées »". Ils avancent également qu'à l'époque, ils ne comprenaient pas les lois concernant la propriété. Au cours des dernières années, et suite notamment aux informations diffusées à ce sujet sur les chaînes télévisées nationales ${ }^{10}$, ils ont été amenés à considérer l'expropriation de leurs terres non seulement comme étant « injuste » mais également « illégale ».

Depuis l'ouverture du «parc du grand banian», et particulièrement depuis la croissance du tourisme domestique à Yangshuo, de nombreux parcs et sites touristiques ont été construits autour de la ville, soit par les autorités locales, soit par des joint-ventures (avec des investisseurs de Taïwan, Hong Kong ou Singapour), soit par des entrepreneurs urbains. Dans la plupart des cas, des conflits relatifs à l'expropriation des terres ainsi qu'au montant et à la nature des compensations ont éclaté entre les habitants locaux et les responsables des parcs. Ces expropriations montrent comment les habitants locaux sont de plus en plus souvent mis à l'écart du développement du tourisme, comme c'est le cas ailleurs en Chine. (Notar 2006 : 115-26 et Nyìri $2006: 46$ ).

9. Ces citations ont été recueillies lors des entretiens réalisés au village en mars 2007.

10. Plusieurs villageois ont cité la chaîne nationale CCTV 12, chaîne intitulée « Société et la Loi » en chinois et qui a pour but de promouvoir la connaissance de la loi en mettant en scène la résolution de conflits juridiques. 
Parallèlement à la croissance du tourisme domestique, Yangshuo a connu un afflux important d'entrepreneurs citadins et étrangers. Une majorité croissante d'hôtels, de restaurants, et autres commerces destinés aux touristes est maintenant gérée par ces derniers. On fait appel à une main-d'œuvre urbaine et qualifiée (issue des écoles de tourisme ou d'hôtellerie) et la population locale, peu éduquée, se voit cantonnée à des emplois subalternes. L'implantation d'entrepreneurs extérieurs est également facilitée par le clientélisme et la corruption qui règnent dans le gouvernement local plus orienté vers l'accueil de ces entrepreneurs que vers le bien-être de la population. Quant à la majorité des habitants de Yangshuo, ils se voient exclus de la manne touristique du fait de leur manque de capitaux économiques, culturels et politiques.

\section{Visiteurs et visités : un jeu de miroirs}

De plus en plus souvent, l'image de Yangshuo perçue par les touristes chinois n'est pas celle véhiculée par les habitants locaux, mais une image médiatisée par les acteurs citadins et internationaux qui contrôlent les infrastructures et les sites touristiques. Les sites, adaptés au « regard» des touristes citadins reflètent plutôt leurs désirs que les réalités vécues par les habitants locaux. L'une des conséquences de cette tendance est «l'ethnicisation » de l'image de Yangshuo. Nous avons vu que la province de Guangxi où se situe Yangshuo, est désignée depuis 1958 comme une « Région autonome zhuang ». Les Zhuang, qui représentent $33 \%$ de la population totale, sont concentrés dans la moitié occidentale de la région autonome. Mais, en raison de ce statut de région autonome et de l'association déjà évoquée avec le film Liu Sanjie inspiré d'une légende zhuang, la ville éveille un sentiment d'exotisme pour de nombreux touristes, bien que cette minorité soit totalement absente dans les zones concernées. Ce sentiment est mobilisé et renforcé par les promoteurs touristiques qui tentent de tirer profit de l'attrait qu'exerce cet exotisme.

C'est le cas pour le parc « La source aux pêchers hors de ce monde » (Shiwai taoyuan), situé sur la route principale entre Guilin et Yangshuo. Créé en 1997 par un joint-venture entre un entrepreneur taïwanais et le gouvernement local, il constitue l'un des sites incontournables du tourisme à Yangshuo, attirant jusqu'à un million de visiteurs par an. Il s'agit d'un parc à thème inspiré du texte " Note sur la source aux fleurs de pêcher » (Taohua yuan ji), de Tao Yuanming, poète célèbre du $4^{\mathrm{e}}$ siècle de notre ère. Ce texte relate l'histoire d'un pêcheur qui, passant à travers une grotte, se retrouve dans un paradis perdu où les habitants primitifs et accueillants vivent en totale autarcie, inconscients de l'essor et du déclin des dynasties impériales dans le monde extérieur. Il s'agit de l'un des textes les plus célèbres de la littérature chinoise, connu de tous, et qui a nourri l'imaginaire de générations de voyageurs lettrés désireux de retrouver cet eden ${ }^{11}$. Tao Yuanming n'a aucun lien avec le Guangxi, et la décision de construire ce parc dans ce lieu reflète surtout l'opportunisme et le sens des affaires de l'entrepreneur qui a saisi la résonance que ce texte pouvait avoir dans l'imaginaire de touristes urbains modernes.

11. À ce sujet voir la condamnation par Shen Congwen, auteur de la première moitié du $20^{\mathrm{e}}$ siècle, de la naïveté des voyageurs lettrés qui, encore dans les années 1930, imaginaient pouvoir retrouver la source aux pêchers (Shen 2004 [1936] : 10-11). 


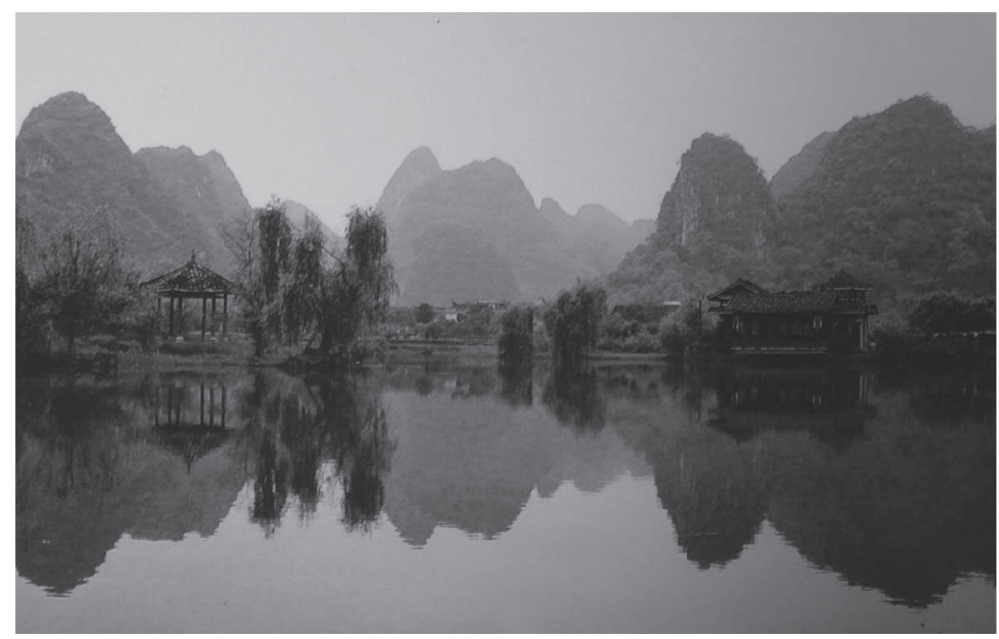

Le parc de Shiwai taoyuan et le paysage environnant, avril 2007 (C) Timothy Leicester

La visite, qui s'effectue en groupe, accompagné d'une jeune guide habillée en costume folklorique, est organisée en trois étapes. La première consiste en un voyage en barque à travers une grotte submergée à la sortie de laquelle se trouve un village en bambou où des acteurs, habillés en peau d'animal, incarnent les membres d'une " tribu primitive » (yuanshi buluo $)^{12}$. Mettant en scène des « rituels ancestraux» les « femmes primitives » lancent des regards amoureux vers les touristes masculins et le guide apprend aux visiteurs comment dire « je t'aime» dans le langage (inventé) de la tribu. Cette première partie de la visite invite les touristes à vivre l'expérience du pêcheur dans l'histoire de Tao Yuanming et à découvrir par eux-mêmes le paradis perdu de la source aux pêchers.

Une fois revenus à terre dans le «monde moderne », le circuit se poursuit avec une visite guidée d'un village "ethnique» inspiré de l'architecture vernaculaire Dong, nationalité minoritaire qui habite dans les hautes-terres à la frontière entre les provinces de Guangxi, Guizhou et Hunan. Le groupe est accueilli à l'entrée du village par des femmes Dong en costume de fête, et est invité à boire des verres d'alcool de riz. Cet accueil est à la fois une réinterprétation de rituels effectivement pratiqué dans les villages Dong (voir Schein 1997 : 70) mais il est également une mise en scène du texte de Tao Yuanming dans lequel les habitants de la grotte tuent un poulet et versent de l'alcool pour accueillir le pêcheur. Les visiteurs assistent et participent aux danses «traditionnelles », puis à la cour amoureuse qui consiste à lancer et à attraper des ballons en coton cousu. Ensuite, il y a des démonstrations de l'artisanat de plusieurs minorités ethniques du Sud-ouest. Les personnes qui «peuplent » ce village, effectivement issus de ces diverses minorités $\mathrm{du}$ Sud-ouest, logent sur place. La dernière partie du voyage se poursuit par la visite de la reconstitution d'une maison de lettré chinois autour de jardins paysagers et de cours intérieures. Le guide explique la vie de Tao Yuanming et souligne les similitudes entre sa retraite à la campagne, et celles des touristes modernes qui ont besoin d'échapper à la « pollution » des villes.

12. Je reprends ici les termes employés par les guides au cours de la visite. 
En s'inspirant du texte de Tao Yuanming, ce site initie les touristes au tourisme lettré pré-moderne et à l'esthétique paysagère chinoise, dont Tao est l'un des précurseurs. On les invite à s'imaginer comme les descendants modernes des lettrés d'antan. Quant aux personnes issues des minorités ethniques dans le parc, elles sont présentées comme étant l'équivalent moderne des " primitifs" réfugiés dans la grotte. Leurs « coutumes ancestrales » et leur travail artisanal sont exposés comme un contrepoint aux professions modernes des touristes. Le parc semble mettre en scène la tendance, évoquée par MacCannell (1976 : 91), des touristes contemporains, aliénés par leur propre travail, à idéaliser celui des autres. Mais il démontre une tendance plus spécifiquement chinoise qui consiste à «primitiviser» les minorités ethniques. Le texte de Tao Yuanming évoque le mythe, très répandu à travers l'histoire de la pensée chinoise, d'un « âge d'or », situé avant l'avènement des bureaucraties impériales. Au cours des siècles, de nombreux voyageurs chinois ont cru voire dans les mœurs hétérodoxes des peuples frontaliers les vestiges de cet âge d'or ${ }^{13}$. À l'époque moderne c'est la pensée évolutionniste de Marx qui renforce la représentation des ethnies minoritaires comme étant « primitives » ou représentatives du passé (Gladney 2004 : 60). Par opposition, la majorité han se situe vers le haut de cette chaîne d'évolution sociale. En observant les minorités ethnique dans le parc, présentées par les guides comme étant des « fossiles vivants», les touristes renforcent leur propre sentiment d'appartenir à la société « moderne».

L'image exotique projetée sur les minorités ethniques par les touristes urbains est symptomatique de ce que Louisa Schein, dans ses travaux sur la province voisine de Guizhou, a qualifié d' « orientalisme interne » (Schein 1997) en référence à la célèbre thèse d'Edward Saïd (1978). Par ce processus, les minorités ethniques sont érigées en un "Autre interne » qui est à la fois un objet de désir, et une image qui rassure et renforce le sentiment de supériorité des observateurs. À Guizhou, comme à Yangshuo, cet orientalisme interne est constitué de thèmes similaires : l'érotisation des femmes, et le rapprochement des ethnies minoritaires avec l'enfance et la nature. Ces thèmes soulignent la virilité, la maturité et le niveau supérieur de la culture dominante et sont constitutifs de l'identité chinoise moderne (Schein 1997 : 90). Focaliser le regard sur l'Autre, idéalisé et représentatif du passé, est également une manière de faire abstraction des problèmes contemporains.

Pour les touristes urbains, il est en effet plus facile de projeter des idéaux sur un «Autre » exotique (les ethnies minoritaires) à l'intérieur du parc que sur les paysans locaux dont le statut dans l'imaginaire urbain est beaucoup plus ambigu. Beaucoup de touristes appartiennent à la « génération perdue » concernée par les mouvements d'envoi massif de jeunes urbains instruits à la campagne entre 1968 et 1980 (le mouvement de xiaxiang). Enthousiastes au départ, leurs idéaux maoïstes se sont vite évanouis une fois confrontés aux rudes conditions de vie et au bas niveau culturel des paysans (Bonnin 2004 : 243-326). Cette expérience traumatique a profondément marqué l'esprit de cette génération et leurs relations avec la paysannerie. Ces touristes n'hésitent pas à qualifier mes propres recherches de terrain dans les villages comme du «xiaxiang » avec toutes les connotations négatives que cela comporte. La mentalité modernisatrice, encouragée par l'État, fait que même pour les touristes qui n'ont pas connu directement ce mouvement,

13. En ce qui concerne Guilin c'est le cas notamment de Fan Chengda qui a fait l'éloge des de « la pureté et de l'antiquité des mœurs locales » (Fan 1991 [1174] : 1). 
les réalités des campagnes sont trop souvent vécues comme un triste rappel de l'arriération du pays. Il est alors préférable, comme l'un des guides du parc me l'a fait remarquer, de visiter la version édulcorée et exotique de la vie villageoise présentée à Shiwai taoyuan que les « vrais » villages « arriérés » (luohou).

La population locale est presque totalement exclue du succès du parc. Les guides sont d'origine urbaine, diplômés des écoles de tourisme, et les représentants des minorités ethniques viennent d'autres régions. Les seuls postes ouverts à la main-d'œuvre locale concernent le nettoyage et l'entretien du site. Ne constituant pas des objets touristiques désirables en eux-mêmes, les paysans et leurs contrées sont réduits à de simples éléments du paysage, consommé à distance par le regard touristique. Il est doublement difficile pour eux de s'approprier ce regard puisque, si on les considère comme faisant partie du paysage, les paysans eux-mêmes sont nombreux à ignorer cette notion, issue d'une esthétique de l'espace culturellement acquise (Roger 1997: 64-82) que beaucoup ne maitrisent pas. Bon nombre de mes interlocuteurs paysans ne «voyaient» pas le paysage (fengjing), et certains ne connaissait pas le sens même du mot. En effet, dans ces villages, c'est le fengshui, ou géomancie, et non pas l'esthétique paysagère qui constitue la base des rapports à l'espace et à l'environnement. Ceci démontre une fois de plus que le paysage appartient aux agents qui en ont la gestion (c'est-à-dire les autorités et investisseurs) ainsi qu'aux touristes qui le consomment, et non aux habitants locaux. Si les réglementations qui régissent les mingsheng définissent le paysage comme étant une « ressource» (ministre des Affaires d'État, 2006), dans la pratique, seuls ceux qui le voient sont capables d'en disposer. Il s'agit alors d'une autre forme d'appropriation de l'espace rural par le regard touristique ${ }^{14}$.

Certains habitants de deux villages voisins du parc ont tout de même mesuré cette injustice, et ont récemment réclamé des dédommagements aux gérants du parc en arguant que leurs villages et leurs champs faisaient partie des paysages autour du parc (Sun et Bao 2007 : 87). À leurs yeux, puisque ces paysages sont appréciés par les touristes et constituent l'un des attraits du parc, il est normal que ce dernier leur verse une somme d'argent pour leur rôle dans leur entretien. Ils ont également fait valoir que ces paysages sont le fruit du dur labeur de leurs ancêtres. À plusieurs reprises, des villageois ont participé à des actions collectives devant le siège administratif du parc jusqu'à ce que ce dernier, soucieux de garder de bonnes relations avec ses voisins, ait cédé à leurs demandes et se soit engagé à privilégier les habitants de ces deux villages pour les emplois à l'intérieur du parc.

Le village de Chuanyan à Yangshuo, évoqué plus haut, constitue un autre cas où des villageois ont réussi à s'approprier partiellement le regard touristique. Malgré le sentiment d'injustice à l'égard des autorités locales à la suite de la construction du parc, les villageois ont longtemps profité du tourisme en vendant des souvenirs, de la nourriture, ou des voyages en radeau. Les revenus de ces activités ont largement compensé les pertes provoquées par l'expropriation de leurs terres et a permis à de nombreuses familles d'envoyer leurs enfants à l'école et à l'université dans les grandes villes et même à l'étranger. Cependant, les relations entre la direction du parc et les villageois se sont rapidement dégradées en 2002 quand ces derniers se sont vus interdire de faire du commerce à l'intérieur du

14. Les mêmes réglementions interdisent la construction de tombeaux dans les paysages définis comme mingsheng alors que la situation des tombeaux est un des éléments fondamentale de la géomancie chinoise et un moyen par lequel les individus maitrisent leur espace. 
parc. Cette interdiction a été renforcée par l'envoi d'un groupe d'hommes musclés qui a confisqué les radeaux et les a brûlés devant le village. Les autorités du parc ont déclaré que, désormais, si les habitants voulaient conduire des touristes en radeau, ils devaient le faire seulement par l'intermédiaire du parc, touchant en retour un salaire journalier.

Les villageois, pour lesquels les revenus du tourisme étaient devenus indispensables, ont perçu ceci comme une grave atteinte à leurs moyens de survie. Sous la direction du chef du comité villageois, les habitants de Chuanyan ont fondé une entreprise dénommée « La compagnie de Liu Sanjie », d'après le personnage du film éponyme, dont tous les villageois sont actionnaires. Ils ont également aménagé une partie du village en site pittoresque comprenant un musée d'anciens outils agricoles, qu'ils ont nommé «La maison de Liu Sanjie », et une grotte aménagée. Ils proposent également des voyages en radeau en compagnie d'une chanteuse habillée en costume folklorique, qui fait la sérénade aux touristes lorsqu'ils admirent le paysage et boivent le thé. Pour aménager la grotte ils ont fait appel aux hommes âgés du village ayant reçu une éducation classique avant la révolution communiste. Ceux-ci ont nommé les différentes parties de la grotte selon la tradition lettrée et ont inscrit des poèmes sur les murs, en l'occurrence des poèmes de Mao Zedong, le « poète des paysans ».

Défiant les autorités du parc, les villageois ont ouvert leur propre entrée du parc à cinquante mètres de l'entrée «officielle» au nouvel An 2003. Malgré plusieurs mois d'affrontements musclés avec la direction du parc soucieuse de protéger ses revenus, celle-ci a finalement cédé sur l'accès au banian. Les conflits entre les deux parties ne sont pas prêts de finir, mais quelque soit leur dénouement, le cas de Chuanyan montre la possibilité, aussi réduite soit-elle, que les paysans ont de s'approprier le regard touristique et de s'en servir pour leur profit. Pour ce faire, les villageois de Chuanyan ont bénéficié d'une longue période de socialisation auprès des touristes durant laquelle ils ont intériorisé les discours et les attentes de la culture dominante. D'autre part, les connaissances acquises par les personnes vivant à l'extérieur du village leur ont permis de mieux s'organiser, en adaptant les structures collectives léguées par la période maoïste, pour faire face aux pressions des autorités locales.

\section{Conclusion}

Le cas de Chuanyan nous rappelle ces Balinais observés par Michel Picard qui «ne sont pas les objets passifs du regard touristique, mais des sujets actifs qui construisent des représentations de leur culture à l'usage des touristes, des représentations fondées à la fois sur leurs propres systèmes de références et sur leur interprétation du désir des touristes. » (2001 : 120). Ou encore ces villageois de Guizhou observés par Tim Oakes qui, dans la rencontre touristique, «construisent leur propre subjectivité moderne » (1998: 48). Les villageois de Chuanyan et d'autres lieux autour de Yangshuo ne sont pas passifs face au développement du tourisme et emploient diverses stratégies individuelles et collectives pour en bénéficier. Cependant, comme des chercheurs chinois l'ont récemment souligné, les communautés balinaises possèdent « un pouvoir de négociation beaucoup plus fort que celui de paysans chinois » (Sun et Bao 2007 : 15). Selon eux, le tourisme rural en Chine est « une forme de connivence entre dominants » (Ibid. : 18), entre investisseurs et gouvernements locaux. La possibilité qu'ont les paysans autour de Yangshuo de «construire des représentations de leur culture » est très limitée. Les habitants de Chuanyan doivent ainsi occulter leur propre système de référence (par exemple la sacralité du banian), pour se conformer au regard paysager et orientalisant des touristes urbains. 
Le pôle de la modernité pour ces paysans se situe ailleurs que dans les campagnes, et ce n'est pas dans la « rencontre » touristique, trop inégale, qu'ils peuvent négocier leur « subjectivité moderne ». Cela se reflète dans leurs propres formes de mobilité vers les villes, où le désir de "voir le monde », c'est-à-dire les grands centres urbains, et aussi important que les motivations économiques.

Dans ce contexte, où les touristes à Yangshuo ne sont accueillis qu'avec l'image de leurs propres désirs, il est difficile, voir impossible, de cerner ce qui est « authentique ». Il est évident qu'une préoccupation pour l' « authenticité » est au cœur de la démarche d'un grand nombre de touristes occidentaux. Un couple de touristes français, qui faisaient partie d'un groupe touristique que j'ai accompagné récemment en Chine m'a expliqué leur choix de voyage ainsi : «Nous aimons les pays authentiques avec des gens authentiques, mais aujourd'hui il faut aller de plus en plus loin pour en trouver ». C'est un exemple frappant des contradictions de la modernité qui « permet d'imaginer une vie authentique, alors que paradoxalement elle met en mouvement des forces qui rendent cette authenticité de plus en plus difficile à atteindre » (Oakes 1998 : 24). Si l'intérêt porté par les touristes chinois aux ethnies minoritaires et à leurs méthodes de fabrication artisanale semble démontrer le même souci d'authenticité, nous avons également vu que cet intérêt à des racines plus spécifiquement chinoises. Dans la pratique, la grande majorité des touristes chinois ne se soucient pas de l' « authenticité » de leurs expériences touristiques. Des guides chinois se disent conscients de cette différence, et doivent en conséquence adapter leur travail selon leur clientèle. Pour eux, ces différences s'expliquent par la relative nouveauté du tourisme en Chine et du fait que les touristes chinois n'ont pas eu le temps d'affiner leurs goûts pour différencier ce qui est « authentique » de ce qui ne l'est pas. Pour notre part, ces différences relèvent de la tradition du tourisme lettré dans laquelle il est accepté, et même nécessaire à l'appréciation d'un site, qu'il soit reconnu et médiatisé par un ensemble de références littéraires et culturelles. Ce n'est pas l'authenticité qui est valorisée mais l'embellissement d'une réalité par l'artifice de la civilisation qui est, depuis ses origines, au cœur du concept du site dans le tourisme chinois. D'autre part, la modernité continue à exercer un fort pouvoir d'attraction sur les touristes chinois, qui n'en sont pas suffisamment désenchantés pour vouloir se lancer dans une quête d'authenticité.

Aujourd'hui, certains en Chine rejettent le tourisme de masse et ridiculisent le « tourisme paysager traditionnel » pour lequel « la Nature est perçue comme un objet de consommation, et la relation entre le touriste et le paysage est réduit à une transaction commerciale » (Shen 2006 : 10). Le même auteur, un défenseur de l' « éco-tourisme » («shengtai lü̈ou»), critique le surdéveloppement des sites touristiques à Guilin et Yangshuo, transformant une citation souvent associée à la région: «les plus beaux paysages sous le ciel » en « les paysages les plus artificiels sous le ciel » (shanshui jia tianxia). Cette dichotomie entre paysages « artificiels » et « authentiques » est étrangère à la tradition paysagère chinoise et montre l'influence du regard paysager romantique. Si on doit applaudir sa volonté de réduire les effets néfastes du surdéveloppement touristique et s'il est également vrai que l'influence du tourisme lettré a des implications particulières pour le développement du tourisme en Chine, il n'y pas de raison de croire que l'idéalisation de la nature sauvage soit mieux adaptée pour réduire ces effets néfastes ${ }^{15}$. Le contrôle, par

15. Il suffit de voir comment cet idéal a été utilisé dans d'autres contextes de fortes inégalités sociales par des groupes dominants pour exproprier les terres des habitants ruraux (Giraut et al. 2005). 
des réseaux économiques et politiques, d'un tourisme rural dont les ruraux eux-mêmes sont largement exclus s'inscrit dans un contexte plus large de fortes inégalités sociales, dont il est difficile d'imaginer qu'elles ne soient perpétuées et renforcées par le regard touristique, fût-il « romantique » ou « lettré ».

\section{Références citées}

Anagnost, Ann S., 1997. National Past-Times: Narrative, Representation and Power in Modern China. Durham: Duke University Press.

Bonnin, Michel, 2004. Génération perdue : le mouvement d'envoi des jeunes instruits à la campagne en Chine, 1968-1980. Paris : EHESS.

Breidenbach, Joana et Pál Nyìri, 2007. “'Our Common Heritage', New Tourist Nations, Post-'Socialist' Pedagogy, and the Globalization of Nature”, Current Anthropology, 48 (2), pp. 322-330.

Brook, Timothy, 1998. The Confusions of Pleasure: Commerce and Culture in Ming China. Berkeley: University of California Press.

CAHILl, James, 1992. "Huang Shan Paintings as Pilgrimage Pictures" in Susan Naquin and Yu Chun-Fang (eds), Pilgrims and Sacred Sites in China, pp. 246-292. Berkeley: University of California Press.

Chan, Yuk Wah, 2006. "Coming of Age of the Chinese Tourists: The Emergence of Non-Western Tourism and Host-Guest Interactions in Vietnam's Border Tourism”, Tourist Studies, 6, pp. 187-213.

Cheng, Tiejun et Mark Selden, 1994. "The Origins and Social Consequences of China's Hukou System”, The China Quarterly, 139, pp. 644-668.

Clsa, 2005. "China Outbound Tourism Industry Ready to Boom”, accessible sur www.clsa.com, consulté le 15 mai 2008.

CoHEn, Eric, 1994. "Contemporary Tourism - Trends and Challenges: Sustainable Authenticity or Contrived Post-modernity?", in Richard Butler et Douglas Pearce (eds), Change in Tourism: People, Places, Processes, pp. 12-29. Londres: Routledge.

CNTA (China National Tourism Agency), “2007 nian guonei lüyou jiben qingkuang” (« Résumé général du tourisme interne en 2007 »), accessible sur www.cnta.gov.cn, consulté le 17 mai 2008.

DAVID, Béatrice, 2007. « Tourisme et politique : la sacralisation touristique de la nation en Chine », Hérodote, 125 , pp. 143-156.

Evrard, Olivier, 2006. «L'exotique et le domestique - Tourisme national dans les pays du Sud : réflexion depuis la Thaïlande », Autrepart, 40, pp. 151-167.

FAN, Chengda, 1991 [1174]. Guihai yuheng zhi (Monographie de la prédication et la mesure de la mer d'osmanthes). Pékin: Zhonghua Shuju.

Giraut, Frédéric, Sylvain Girau et Myriam Houssay-Holzschuch, 2005. «La nature, les territoires et le politique en Afrique du Sud », Annales Histoire, Sciences sociales, 60 (4), pp. 695-720.

Gladney, Dru, 2004. Dislocating China: Muslims, Minorities and Other Subaltern Subject. London: Hurst and Company.

Guo, Xiaolin, 2001. "Land Expropriation and Rural Conflicts in China", The China Quarterly, 166, pp. 422-439.

Hutchings, Graham, 1986. "A Province at War: Guangxi during the Sino-Japanese Conflict, 1937-45", The China Quarterly, 108, pp. 652-679.

Ho, Peter, 2001. "Who Owns China’s Land: Policies, Property Rights and Deliberate Institutional Ambiguity”, The China Quarterly, 166, pp. 394-421.

KaHN, Joseph, 2004. “China's Leader Urges Shift in Development to Rural Areas”, New York Times, 4 mars.

Kaup, Katherine Palmer, 2000. Creating the Zhuang: Ethnic Politics in China. Boulder: Lynne Rienner. 
Maccannell, Dean, 1976. The Tourist: A New Theory of the Leisure Class. New-York: Schocken Books.

McKhann, Charles, 2001. "Tourisme de masse et identité sur les marches sino-tibétaines : Réflexions d'un observateur », Anthropologie et Sociétés, 25 (2), pp. 35-54.

Ministre des AfFaires D’État, 2006. "Fengjing mingsheng qu tiaoli” (« Réglementations des sites pittoresques et célèbres »), accessible sur www.gov.cn, consulté le 23 janvier 2007.

Naughton, Barry, 1995. "Cities in the Chinese Economic System" in Deborah S. Davis et al. (eds), Urban Spaces in Contemporary China, pp. 61-89. Cambridge: Cambridge University Press.

NiQuet, Valérie, 1997. “Tourism: Marketing China”, China News Analysis, 1606, pp. 1-10.

Notar, Beth E., 2006. Displacing Desire: Travel and popular culture in China. Honolulu: University of Hawai'i Press.

Nyìri, Pál,

2006. Scenic Spots: Chinese Tourism, the State, and Cultural Authority. Seattle: Washington University Press.

2005. "Scenic Spot Europe: Chinese Travellers on the Western Periphery", accessible sur www.espacesTemps.net, consulté le 13 janvier 2007.

OAKES, Tim, 1998. Tourism and modernity in China. Londres: Routledge.

WTO (World Tourism Organisation), 2005. "Indicators of Sustainable Development for Tourism in Yangshuo China”, accessible sur www.world-tourism.org, consulté le 15 février 2007.

PICARD, Michel, 2001. « Bali: vingt ans de recherches », Anthropologie et Sociétés, 25 (2), pp. 109-127.

Roger, Alain, 1997. Court traité du paysage. Paris : Gallimard.

SAïD, Edward, 1978. Orientalism. New York: Vintage Books.

ScheIn, Louisa, 1997. “Gender and Internal Orientalism in China”, Modern China, 23 (1), pp. 69-98.

SHEn, Congwen, 2004 [1936]. Xiang xing sanji (Ecrits divers sur mes voyages dans le Hunan). Pékin: Renmin wenxue.

SHEn, Xiaohui, 2006. "Shengtai lüyou: Yao jujue shehua yu rengong hua” (« Tourisme écologique: il faut éviter l'extravagance et l'artifice »), Ziran zhiyou tongxu, 46, pp. 10-12.

STRASSBERG, Richard E., 1994. Inscribed landscapes: travel writing from imperial China. Berkeley: University of California Press.

Sun, Jiuxia et Bao JigANG,

2006. "Shequ canyu de lüyou renleixue yanjiu: Yangshuo Shiwai Taoyuan anli” (« Étude d'anthropologie du tourisme sur la question de la participation communautaire: Le cas du 'Parc de la source des pêchers hors de ce monde’ à Yangshuo »), Guangxi minzu xueyuan bao, 26 (1), pp. 82-90.

2007. « Differences in community participation in tourism development between China and the West », Chinese Sociology \& Anthropology, 39 (3), pp. 9-27.

TAn, Chee-Beng, Sydney Cheung et Yang Hui (eds), 2001. Tourism, Anthropology and China. Bangkok: White Lotus Press.

THIREAU, Isabelle et Hansheng WANG (éds), 2001. Disputes au village chinois : formes du juste et recompositions locales des espaces normatifs. Paris: EHESS.

URRY, John, 1990. The tourist gaze. Londres: Sage.

WANG, Qingsheng,

2006. Xianjing Yangshuo, menghua jiayuan (Paysages fantastiques de Yangshuo, pays natal des rêves). Guilin: Lijiang Chubanshe.

2004. Yangshuo daoyou (Guide de Yangshuo). Yangshuo : Bureau du tourisme. 


\section{Conflits et enjeux identitaires dans le tourisme rural à Yangshuo}

Wu, Yongyun, 1997. “ “Wenhua Xiaxiang’ qianyi” (« Remarques préliminaires sur 'l’envoi de la culture dans les campagnes’ »). Anhui Ribao, 21 mars.

Xu, Gang, 1999. Tourism and Local Economic Development in China: Case Studies of Guilin, Suzhou and Beidaihe. Richmond: Curzon.

Xu Xinxian, 1920 [1673]. Yangshuo Xianzhi (Monographie locale de Yangshuo). 4 vol., manuscrit.

ZhANG, Jiaxin, 2004. Mingdai de lüyou shenghuo (La vie touristique de la dynastie Ming). Yilan: Mingshi yanjiu xiaozu. 